(C) 1977 IEEE. Personal use of this material is permitted. However, permission to reprint/republish this material for advertising or promotional purposes or for creating new collective works for resale or redistribution to servers or lists, or to reuse any copyrighted component of this work in other works must be obtained from the IEEE.

IEEE Transactions on Nuclear Science, Vol.NS-24, No.3, June 1977

\title{
BETTER DYNAMIC CLOSED LOOP CONTROL OF THE PSB RF ACCELERATING SYSTEM
}

G. Gelato and L. Magnani

PS Division, CERN, Geneva, Switzerland

\section{Summary}

The introduction of pulse-to-pulse modulation") (PPM) in the Proton Synchrotron Booster (PSB) requires that a large number of settings be changed on a pulse-topulse basis, imposing a very heavy load on the control system and the operators. Many systems, originally not designed for this type of operation, have to be modified either to accept new settings every cycle (values to be adjusted by the operators, switching to be performed by the control system), or to be self-adaptive, i.e. autoratically adjusting to the required conditions.

The second approach seems preferable, if feasible without excessive increase in complexity or decrease in reliability. In some cases it was actually possible to reduce the complexity and increase the reliability.

This paper deals with the modifications in the beam control system. While reviewing the system's design, additional improvements were found to be possible at moderate cost: they were also, or will be, introduced, and are mentioned accordingly. Mention is made also of a relatively simple method of longitudinal stabilization which has been tested at the PSB.

\section{Radial Control}

In each ring, the radial error signal $\Delta r$ is derived from four of the beam observation pick-up electrodes ${ }^{2}, 3$. They provide $\Delta$ signals proportional to $\Delta \mathrm{r}$ and beam current, and $\Sigma$ signals proportional to current only. The ratio $\Delta / \Sigma$ is proportional to $\Delta r$ only.

In the original system, $\Delta$ and $\Sigma$ went through fixed gain amplifiers, followed by a d.c. restorer to restitute the low frequencies which had been suppressed by the high-pass filtering characteristics of the pick-up (PU) electrodes plus their transmission cables.

The bunch structure was then filtered out by integrators and the signals transmitted to a central room where analogue dividers produced the required $\Delta / \Sigma$ signal for the radial control loop. The dynamic range (about $12 \mathrm{~dB}$ ) was determined by the ratio of the maximum signal not saturating the amplifiers to the minimum required for proper operation of the d.c. restorer. To adapt to various beam intensities, the amplifiers' gain could be changed in steps by remotely controlled relays, with some overlap between ranges to cover pulse-to-pulse jitter and bunching factor variation during the cycle.

Two approaches were considered in order to cope with PPM:

a) Replace the relays with solid state switches (the special relays used, no longer available on the market, would not withstand frequent switching), redesigning the amplifiers to increase the total operating range, which has been substantially expanded since the conception of the system. The control portion of the system (both software and hardware) would have to be modified accordingly.

b) Redesign the amplifiers to cover the complete range without switching. This would involve some form of automatic gain control (AGC), provided one could keep the gain of the $\sum$ and $\Delta$ channels in a fixed ratio for all intensities. No control system would be required in this case.
A solution was found, and adopted ${ }^{4}$, which as a byproduct eliminates the need for d.c. restoration and analogue division. It works on the following principle (see Fig. 1). The $\Delta$ signal is attenuated $(K<1$ ) and inverted, the signal $X \triangle$ is alternatively added to and subtracted from $\Sigma$ by means of gates. $K$ is chosen so that $|\mathrm{K} \Delta|<\Sigma$, even at the largest radial displacement expected $(\simeq 50 \mathrm{~mm})$. The switching frequency $(\simeq 200 \mathrm{kHz})$ is high compared to the cut-off frequency of the radial control loop. An AGC amplifier of gain $\alpha A$ applies the signal to a peak detector which measures the value of $\alpha A(\Sigma+K \Delta)$ and $\alpha A(\Sigma-K \Delta)$ during the gap between bunches (through appropriate choice of the detector polarity). It is easily shown that this is proportional to the d.c. component of the signal, which had been filtered out by the pick-up characteristics. There is therefore no need to recover it through a d.c. restorer and integrator. Synchronous gates store the values of $\alpha A(\Sigma-K \Delta)$ and $\alpha A(\Sigma+K \Delta)$ into two analogue memories. The sum of these two values is compared with a reference voltage $V_{R}$ and the error used to control the main amplifier gain $\alpha A$; we impose therefore $2 \alpha A \Sigma=V_{R}$.

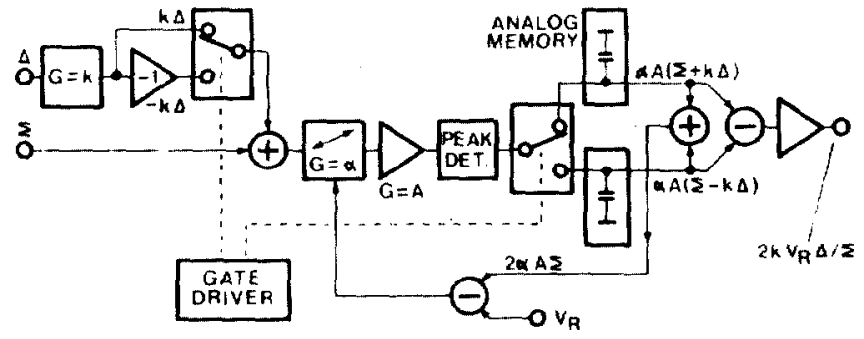

Fig. 1

The difference of the analogue memories' contents is $\alpha A[(\Sigma+\mathrm{K} \Delta)-(\Sigma-\mathrm{k} \Delta)]=\alpha A 2 \mathrm{~K} \Delta=\mathrm{KV}_{\mathrm{R}} \Delta / \Sigma$, i.e. as $\mathrm{K}$ and $\mathrm{V}_{\mathrm{R}}$ are constants, the output signal is proportional to the radial position and independent of the beam current. No further analogue division is required.

The dynamic range of the radial normalizer described is $40 \mathrm{~dB}$, or a 100:1 variation in beam current. A substantial reduction of size and complexity of the system, and an increase in accuracy, reliability and ease of servicing have resulted from the elimination of d.c. restorers, integrators, analogue dividers, attenuators, and remote control.

\section{Phase Control}

In the original system ${ }^{2}$ bunch phase information was derived from PU electrodes, providing a signal proportional to the time derivative of the instantaneous beam current; the RF component of the signal was extracted through a self-tuning filter (TF).

The PU signal is proportional to accelerating frequency, bunching factor, and beam intensity. The first two factors alone give a variation of about $35 \mathrm{~dB}$ during a standard acceleration cycle, occurring mostly during the adiabatic voltage rise after injection $(\approx 400 \mu \mathrm{sec})$. A fast AGC amplifier provided a range of about $20 \mathrm{~dB}$ in front of the TF, which contained an AGC stage of about $25 \mathrm{~dB}$ of control range. As for the radial control, remotely controlled relays provided adaptation to different beam intensities. 
For correct operation, the phase shift of the $\mathrm{TF}+\mathrm{AGC}$ amplifier should be independent of frequency and amplitude. The frequency dependence can be tuned out largely by tailored delay cables; the residual error is slow and is eliminated by the low frequency cut-off of the phase loop amplifier. The amplitude dependence is more critical, because it occurs mostly during the adiabatic rise and is therefore difficult to filter out.

With an expected PPM of the beam intensity of up to $40 \mathrm{~dB}$, the total AGC range of the phase control system had to be increased to about $75 \mathrm{~dB}$, with negligible phase variation.

Full range AGC was considered preferable to attenuator switching, if feasible. Two solutions were found: one consisting simply of an improved AGC amplifier in front of the $T F$, another one involving a double superheterodyne technique with a fixed frequency AGC amplifier, eliminating the TF, a complex piece of hardware, difficult to calibrate and repair.

A working prototype of the second solution has been built, but its refinement has been postponed in favour of the first solution, more straightforward and requiring less development effort. The choice was thus determined by time pressure, but we intend to resume work on the alternative solution at a more leisurely pace.

The new input amplifiers have an AGC range of about $60 \mathrm{~dB}$, with an input-to-output phase variation of $\pm 15^{\circ}$ over the entire amplitude and frequency range ( 3 to $8 \mathrm{MH} \%$ ). The TF has only to provide about $15 \mathrm{~dB}$ of $\mathrm{AGC}$, which improves its phase performance. The over-all performance has not been deteriorated in spite of the $30 \mathrm{~dB}$ increase in dynamic range (from 45 to $75 \mathrm{~dB}$ ).

\section{Radial Correction after Injection}

At the moment of closing the radial loop, the beam radial error is in general not zero. This would produce a switching transient affecting both radial and phase loop, which might trigger instabilities and/or cause proton losses, especially at high beam intensities. The standard remedy was to introduce a time dependent offset voltage in the radial control amplifier adjusted to balance of $f$ the radial error signal at the loop closing time, and to vanish smoothly afterwards, allowing the beam to reach gradually its final position.

It was not possible to compensate in this way the pulse-to-pulse jitter in radial position at injection.

The steering function was generated by the PSB control system. With PPM, this function may require different adjustment from pulse to pulse, due to different injection conditions. This would require more function generator channels and more operator activity.

The difficulty was circumvented by the introduction of an adaptive circuit (Fig. 2 shows the principle of operation) which continuously generates the offset voltage required to keep the error at the input of the radial loop amplifier equal to zero until closing time. At this time, the correction signal is frozen, and then allowed to vanish with a fixed time constant. In this way, the steering function is automatically generated and tailored to the individual position error, coping therefore not only with PPM but also with pulse-topulse jitter.

Referring to Fig. 2, $S_{1}$ and $S_{2}$ act in opposition. The inverter has a gain of -1 , so that with $S_{1}$ closed (radial loop open) the voltage at the summing node is zero; when $S_{1}$ opens, the correction is memorized and allowed to vanish with the $\mathrm{RC} \simeq 4 \mathrm{msec}$, allowing the

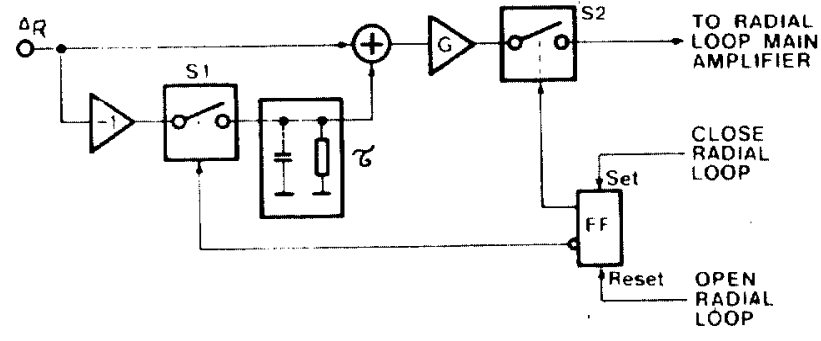

Fig. 2

beam to reach its prescribed position. This result could have been obtained in a number of ways, for example by electrically controlling the gain of the loop amplifier from zero (radial loop open) to its nominal value (loop closed) in a smooth manner. The solution adopted, however, makes the full gain of the radial amplifier immediately available to damp radial perturbations, still suppressing the switching transient.

\section{Frequency Programme}

The accelerating frequency is generated by a voltage controlled oscillator which can be operated in two modes:

a) The control system provides a rough frequency progranme, to which the radial control loop applies the fine corrections required to maintain the correct radial position.

b) The frequency programme is obtained by frequencyto-voltage conversion of the accelerating frequency itself. This is the routine mode of operation. It can be shown that it is equivalent to having an essentially infinite d.c. gain in the radial control loop. With the loop (of which the beam itself is an element) open, the frequency would be undefined: with the loop closed, it is well defined, at the expense of negligible radial offset. As soon as the beam is lost or ejected, the system is immediately switched back to the programmed control, which will continue to act until the next injection.

Pulse-to-pulse modulation of the shape of the magnetic cycle is expected to come into use rather soon. To avoid the need for PPM of the frequency programme in consequence, we $\mathrm{p} l$ an to modify the system by having the oscillator go back automatically and smoothly to the correct frequency for injection, whenever the beam is lost or ejected. We will be able thus to dispense entirely with an external frequency programme.

Evaluation on the machine of two prototypes of the hardware, under construction at present, will begin as soon as possible.

\section{Longitudinal stabilization}

Several forms of coupled-bunch instabilities appear in the PSB at medium to high beam intensities ${ }^{5}, 6$ ) A somewhat crude method of stabilization by "bucket shaking" 2,5) has allowed us to reach and surpass the design intensity in routine operation. A much more sophisticated method involving fast feedback ${ }^{7}, 8$ ) has been introduced recently; besides stabilization, it can provide a very detailed analysis of the various instabilities occurring. We mention here a third method which has provided satisfactory results, namely amplitude modulation of the radio frequency (RF) at the bunch revolution frequency. This causes a bunchto-bunch spread in the synchrotron frequency, thereby 
reducing the coupling between bunches. Figure 3 shows the principle of operation. A sawtooth modulation was applied, resulting in the driving signal of Fig. 4 applied to the power RF amplifier. The corresponding spectrum is shown in Fig. 5 (only the upper sidebands shown), but, due to the filtering effect of the RF cavity, the actual spectrum seen by the bunches was as shown in Fig. 6, corresponding to an almost sinusoidal modulation of $\mathrm{m} \simeq 2.5 \%$. The values used are practical upper limits: with those it was possible to stabilize a total accelerated beam ( 4 rings) of $1.65 \times 10^{13}$ protons, the present PSB record. Other limitations, mainly beam loading, prevented us from testing with higher currents.

In view of the simplicity of this method, the equipment has been kept available as a back-up for the fast feedback system, should the need arise.

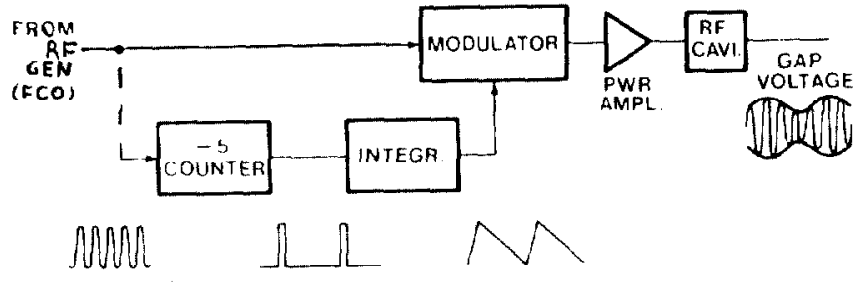

Fig. 3

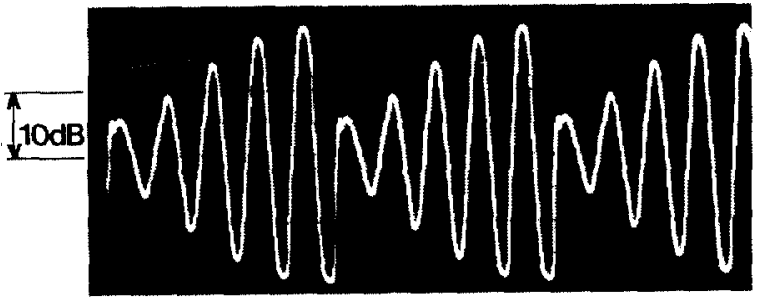

$3 \mathrm{MHz}$

Fig. 4

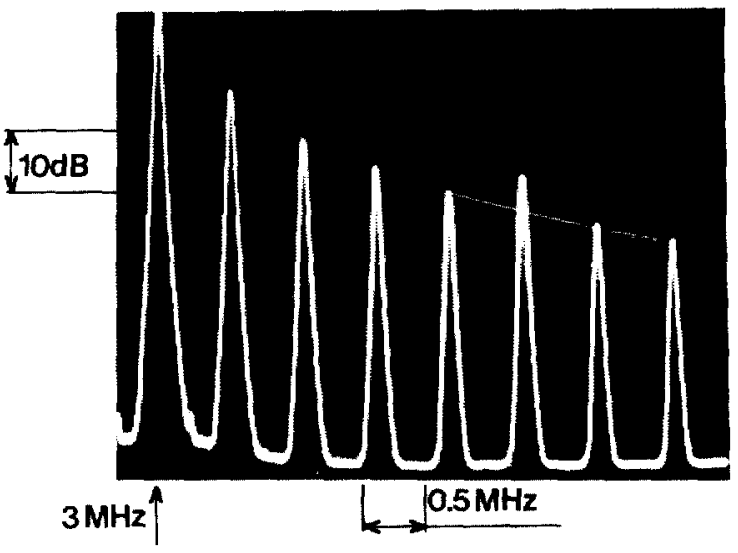

Fig. 5

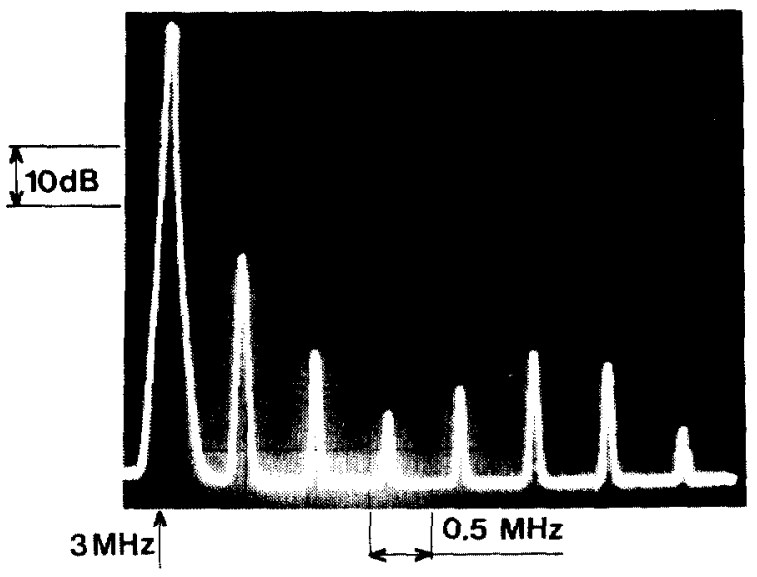

Fig. 6
References

1) The PSB Staff (reported by J.P. Potier), A pulse-topulse modulation of the CERN PS complex, see these proceedings.

2) U. Bigliani, The beam control system for the CERN PS Booster, IEEE Trans. Nuclear Sci. NS-18, 352 (1971).

3) M. Rabany, The orbit measurement system of the CERN $800 \mathrm{MeV}$ PS Booster, Proc. 5th National Particle Accelerator Conf., San Francisco, 1973, IEEE Trans. Nuclear Sci. NS-20, 656 (1973).

4) G. Gelato and L. Magnani, Improved radial pick-up electronics for use over a wide dynamic range, CERN Int. Rep. MPS/Int. BR/75-8 (1975).
5) F.J. Sacherer, A longitudinal stability criterion for bunched beams, Proc. $5^{\text {th }}$ National Particle Accelerator Conf, San Francisco, 1973, IEEE Trans. Nuclear Sci. NS-20, 825 (1973).

6) J. Gareyte, I. Magnani, F. Pedersen, F. Sacherer and K. Schind1, Beam dynamics experiments in the PS Booster, Proc. 6th National Particle Accelerator Conf., Washington, 1975, IEEE Irans. Nuclear $\mathrm{Sci}$. NS-22, 1855 (1975).

7) F. Pedersen and F. Sacherer, Theory and performance of the longitudinal active damping system for the CERN PS Booster, see these proceedings.

8) B. Kriegbaum and F. Pedersen, Electronics for the longitudinal active damping system for the CERN PS Booster, see these proceedings. 